\title{
3D Printed Personalized Guide Plate in the Femoral Head Core Decompression
}

\author{
Liangliang Cheng $(\mathbb{D}$, Xing Qiu, Lei Yang, Chi Xiao, Baoyi Liu, Chukwuemeka Samuel Okoye, \\ and Dewei Zhao
}

Department of Orthopaedics, Affiliated Zhongshan Hospital of Dalian University, No. 6 Jiefang St, Dalian,
Liaoning Province 116001, China

Correspondence should be addressed to Dewei Zhao; zhaodewei2016@163.com

Received 6 August 2020; Revised 27 September 2020; Accepted 7 November 2020; Published 19 November 2020

Academic Editor: Xiaojun Duan

Copyright (C) 2020 Liangliang Cheng et al. This is an open access article distributed under the Creative Commons Attribution License, which permits unrestricted use, distribution, and reproduction in any medium, provided the original work is properly cited.

\begin{abstract}
Objective. To investigate the feasibility of using 3D printed personalized guide plates in core decompression procedures for the treatment of osteonecrosis of the femoral head (ONFH). Methods. The clinical data of 8 patients undergoing femoral head core decompression from January to December 2019 were analyzed retrospectively. Three-dimensional (3D) images of the patients were reconstructed from the CT scan data taken preoperatively. From the data obtained, puncture position, drill hole, and depth were evaluated, and individualized 3D puncture guide plates were designed using Mimics 21.0 software. During the operation, the needle went through the hole of the guide plate, the depth of the drill was controlled, and the obtained bone tissues were sent for pathological evaluation. Intraoperative X-ray and postoperative pathological results were used to evaluate the success of the puncture. Results. The individualized guide plates used for core compression on the 8 patients were well fitted with the anatomic structure of the puncture site, and the direction and depth of the needle insertion were consistent with the preoperative design. The operation time was about 15-22 mins. The position of the decompression tunnel was the same as the designed plate. The postoperative pathology showed necrotic bone tissue. There were no postoperative complications such as infection, bleeding, and fracture. Conclusion. The 3D printed individualized guide plate can simplify core decompression and would make this procedure more accurate, safe, and quick, in addition to obtaining necrotic tissues for pathological examination.
\end{abstract}

\section{Introduction}

Osteonecrosis of the femoral head (ONFH) is a destructive bone condition most commonly affecting young and middleaged patients. ONFH often progresses to femoral head collapse, which in some patients may require total hip arthroplasty (THA) [1-4]. Core decompression is one choice of treatment for early-stage ONFH. Depending on the specific condition, multiple-tunnel decompression or single-tunnel decompression may be adopted. At present, the location of the needle is mainly determined by $\mathrm{C}$-arm fluoroscopy during surgical operation. For young patients with small necrotic areas, repeated fluoroscopy is needed to make the needle reach the ideal position. In recent years, $3 \mathrm{D}$ printed techniques have emerged in the field of medicine due to their high precision, fast construction speed, and on-demand manufacture $[5,6]$.
Orthopedics, oncology, radiotherapy, and other disciplines use $3 \mathrm{D}$ printed personalized guide plates to assist surgery [5-7]. However, the use of this technology in the core decompression procedure has been rarely reported. Recently, the orthopedics department of Affiliated Zhongshan Hospital of Dalian University applied the use of a 3D printed personalized guide plate in core decompression for the treatment of early-stage ONFH and achieved good clinical results.

1.1. Clinical Data. From January to December 2019, the clinical data of eight patients with early-stage ONFH who required core decompression were analyzed retrospectively. The patients included 4 males and 4 females, with ages ranging from 22 to 48 years. Their clinical symptoms included hip pain and limited hip movement. All cases were diagnosed as ONFH (ARCO II) by X-ray and MRI. The study 


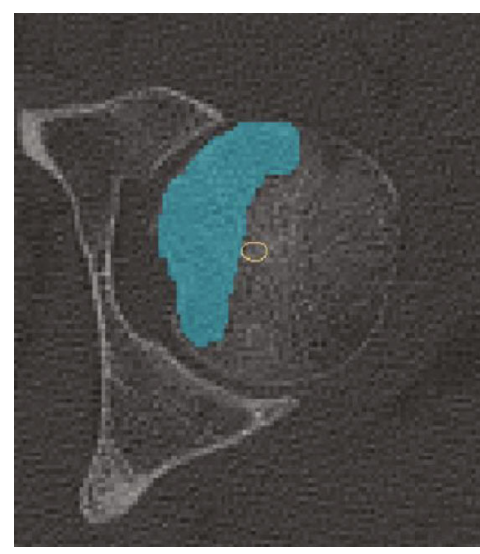

(a)

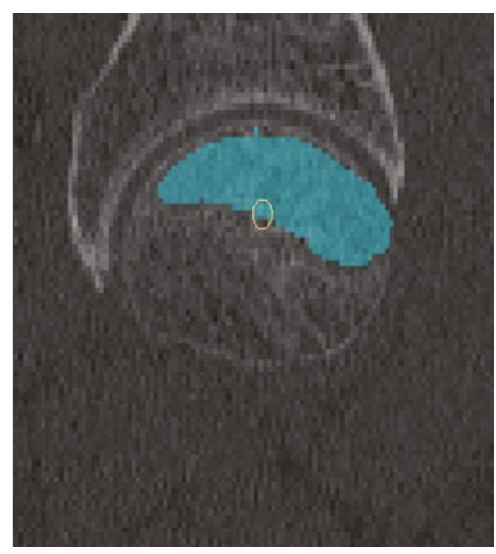

(b)

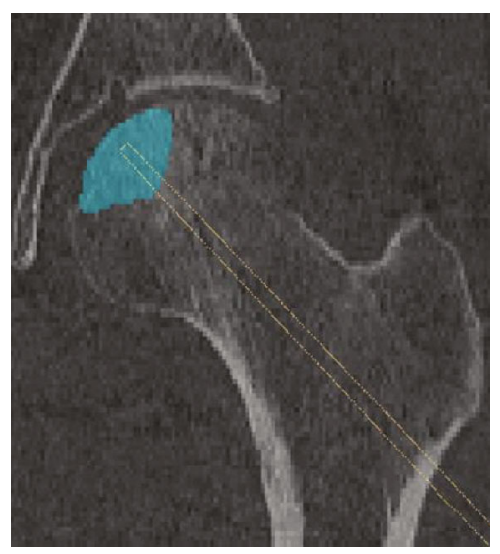

(c)

FIgURE 1: The blue area is the necrotic area of the femoral head, and the yellow line is the location of the decompression pathway: (a) horizontal, (b) sagittal, and (c) coronal.

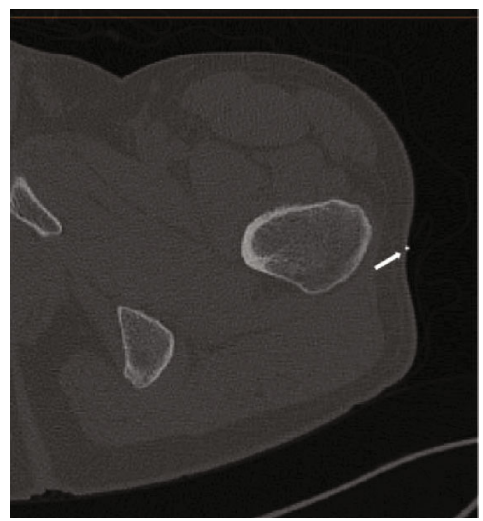

(a)

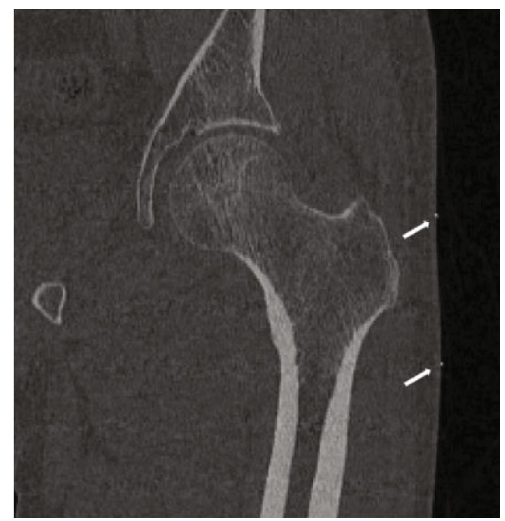

(b)

Figure 2: The little white dots indicated by the white arrow are the metal lines: (a) horizontal and (b) coronal.

was approved by the Ethics Committee of Affiliated Zhongshan Hospital of Dalian University. All patients signed the informed consent form.

\subsection{Three-Dimensional Model of ONFH. CT scan data were} collected before the operation, and three metal wires were used to mark the hip joint before the data were collected. When taking the CT scan, patients were asked to keep their toes close together and their legs straight. After completion of the scan, the CT data was exported to the medical processing software, Mimics 21.0 (Materialise, Belgium). The images were segmented according to the gray value difference amongst the femoral head, skin soft tissue, and the metal wire. The targeted region was segmented with a different threshold, and the $3 \mathrm{D}$ virtual model was reconstructed. The three-dimensional model was optimized, and the osteonecrotic lesion was delineated as the region of interest. The location and shape of the lesion and the relationship between the lesion and the metal lines were highlighted. (Figures 1 and 2).

1.3. Planning of the Decompression Tunnel and Design of Personalized Guide Plates. The center point of the lesion was used as the target point of the decompression tunnel, and the decompression tunnel was planned from the lower part of the greater trochanter of the femur to the center of the necrotic area. After the decompression tunnel was determined, the distance from the skin needlepoint to the decompression target point was measured by the software, and the guide plate was constructed using the software "expansion" function. Taking the decompression tunnel as the center, 5-6 decompression tunnels were designed parallel to it for multitunnel fine needle core decompression. Finally, the redundant parts were removed by the Boolean operation, and the guide plate was customized. The surface of the guide plate in contact with the skin was marked with a trace line on the skin surface of the patient, to easily locate the accurate surface during surgery.

\subsection{D Printing of the Personalized Guide Plate Model. From} Mimics 21.0 software, the guide plate model was exported as a STL file into the 3D printing machine (Stratasys F170) for rapid production of the guide plate. After printing the plate, metal excesses were pruned, the plate was checked on the patient to ensure proper fitting, and the plate surface trace metal line and the trace line marked on the body surface were inspected for consistency. If the comparisons align correctly, the guide plate is sterilized and sealed at low temperature. 


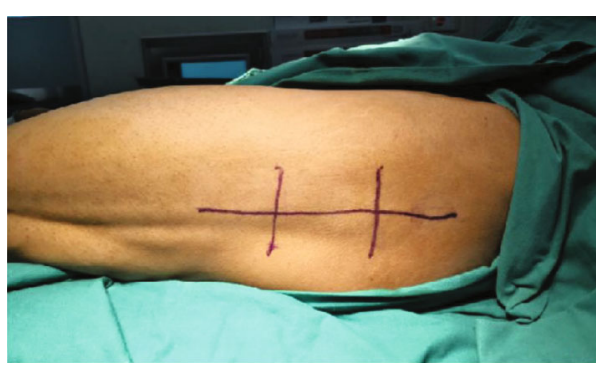

(a)

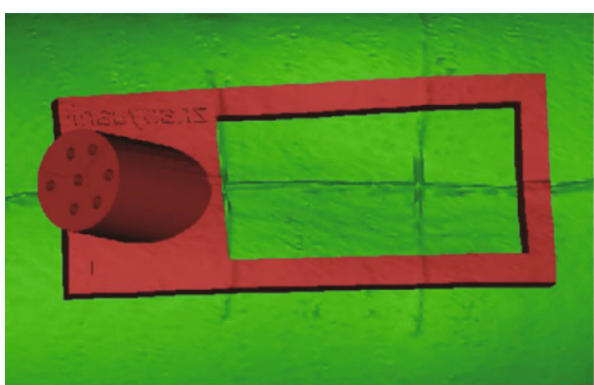

(b)

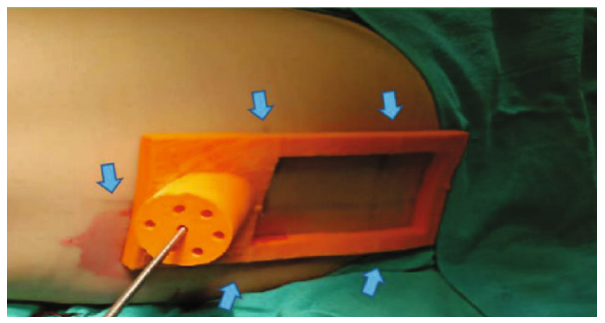

(c)

FIGURE 3: (a) A marker line for the location of the metal line on the body surface; (b) a personalized guide plate designed by the software; (c) the use of a $3 \mathrm{D}$ printed personalized guide plate (the blue arrow indicates the location of the marker line).

1.5. The Application of the Individualized Guide Plate in Core Decompression. After anesthesia, the patient was placed in a horizontal position, and the lower limbs were extended with the toes close to each other, to mimic the same body positioning during CT scan. The marked line on the guide plate is fitted on to the trace line marked on the body surface. Kirschner wire was passed through the guide tunnel in the guide plate, and the needle was slowly penetrated through the predetermined depth. Fluoroscopy was used to confirm the positioning; then, the guide plate was removed. A $1 \mathrm{~cm}$ longitudinal incision was made at the insertion of the Kirschner wire, and trephine was drilled along the Kirschner wire. The Kirschner wire was then pulled out, and necrotic bone was taken out for pathological examination (Figures 3-6).

\section{Results}

The individualized guide plate of the 8 patients fitted properly. The position, direction, and depth of the inserted needle were consistent with the preoperative design. All core decompression operations were completed once, and X-ray fluoroscopy of C-arm confirmed that all the Kirschner wires reached the target point and that the necrotic bone tissues were successfully removed. The operation time was 1522 mins. There were no postoperative complications such as infection, bleeding, and fracture. The histopathologic diagnosis was necrotic bone tissue.

\section{Discussion}

In clinical practice, the treatment of early-stage ONFH is relatively difficult. Core decompression is an effective method to treat early-stage ONFH [8-11]; however, differing results are obtained. Core decompression reduces the pressure in the femoral head through the decompression tunnel, improves the femoral head blood circulation, and at the same time removes the necrotic bone from the focus area, leaving out the healthy bone tissue. Therefore, the location of the decompression tunnel is very important. If the target point of the decompression tunnel can reach the central area of the necrotic focus, it will be more accurate and more effective to remove the necrotic bone. At present, clinicians use intraoperative X-ray fluoroscopy repeatedly to adjust the location of the needle to reach the target point. Repeated punctures can disrupt normal bone tissue and blood circulation within the femoral head and may exacerbate osteonecrosis [12, 13]. Precise puncture decompression is very necessary. At present, navigation technology is used to assist core decompression, which can make the location of puncture decompression more accurate and flexible, but it is necessary to install a positioning device and adjust navigation during the operation which increases the patient limb injury and prolongs the surgical time, and navigation equipment is expensive and so may decrease its usage in the clinics [14].

As 3D printing technology matures [15], existing techniques can be used to design a 1:1 model with a prototype based on the patient's CT data. Complete preoperative planning can be made, and a drill approach can be simulated with a computer. Finally, a personalized 3D printed decompression guide plate is made using $3 \mathrm{D}$ printing technology, which improves the accuracy and efficiency of core decompression.

There are many kinds of 3D printed personalized guide plates used during orthopedic operations [4-6]. They are applied to the bone surface, but not to the surface of the skin, because of the mobility of the skin and soft tissue. The position of the guide plate changes when the body position is changed or pulled apart. Therefore, ensuring the proper positioning of the guide plate is the key to this kind of operation. In this study, before CT scan, 3-4 metal wires were fixed to the thin lateral soft tissue of the greater trochanter of 


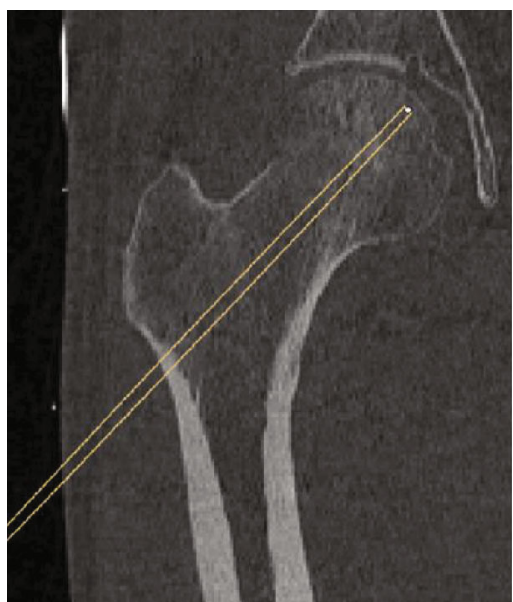

(a)

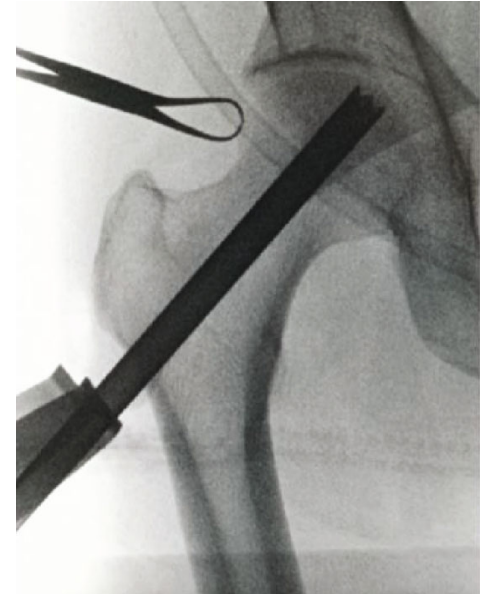

(b)

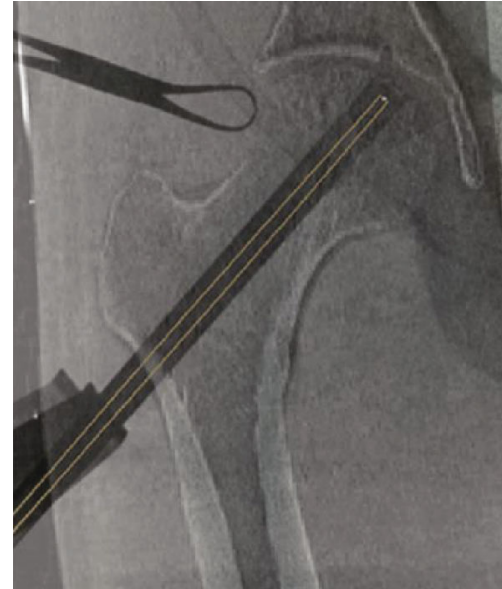

(c)

Figure 4: The preoperative design and intraoperative X-ray film of the decompression tunnel in coronal view (anteroposterior view): (a) the location of the decompression tunnel designed in the software; (b) the X-ray film of the decompression tunnel during the operation; (c) the overlapping of $(\mathrm{a}, \mathrm{b})$, which shows the consistency of the intraoperative and preoperative design.

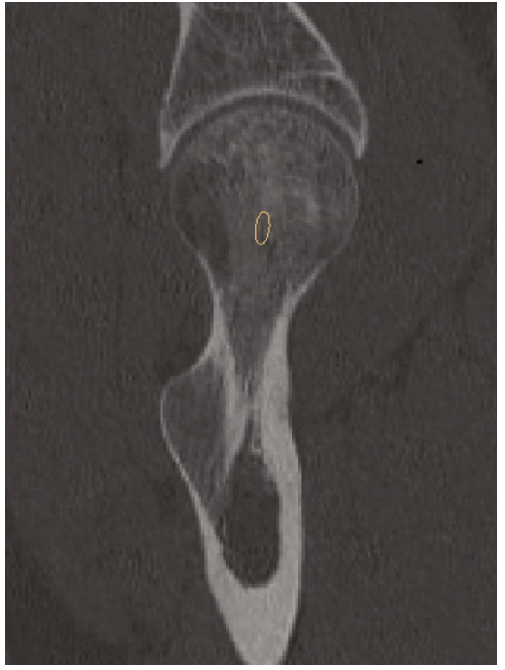

(a)

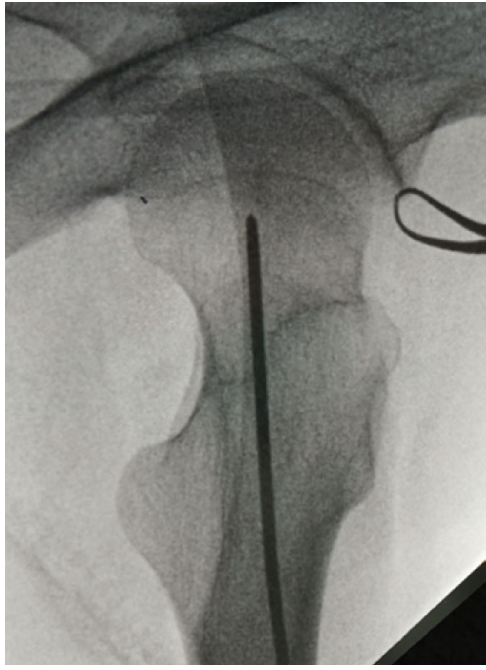

(b)

Figure 5: The preoperative design and intraoperative X-ray film of the decompression tunnel in sagittal view (lateral view): (a) the location of the decompression tunnel designed with the software; (b) the X-ray film of the decompression tunnel during the operation.

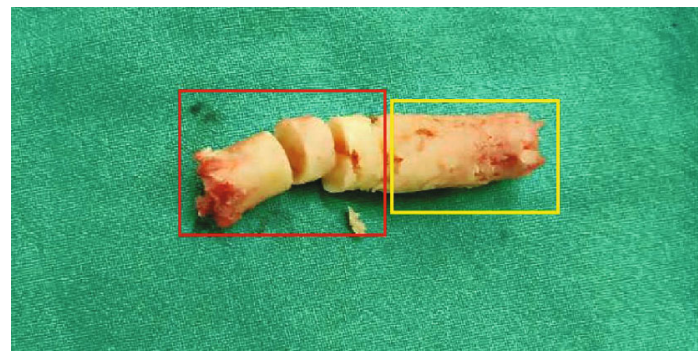

FIGURE 6: The bone tissue was removed through core decompression. The red frame area is necrotic bone tissue, and the yellow frame area is healthy cancellous bone tissue.

the femur as a body surface marker, and the range was as wide as possible; it is important to keep the patient's position during preoperative CT examination consistent with that during intraoperative core decompression, in order to ensure that the appearance and tension of the patient's skin and soft tissue are as consistent as possible. The insertion point at the lateral femur was designed to be $1 \mathrm{~cm}$ below the greater trochanter where the cortical bone is thickening. The insertion point of Kirschner's needle can easily penetrate the cortical bone in this position, thus reducing the deviation of the insertion point caused by sliding of Kirschner's needle. During surgery, the guide plate is gently attached to the trace line marked on the skin surface in a gentle manner to avoid deformation of the skin and soft tissue, which may lead to change of position of the puncture tunnel. Finally, the Kirschner wire with a sharp tip is used for the operation, to ensure that there is no resistance when penetrating the skin and soft tissue and also to avoid error caused by the deformation of Kirschner's needle. For fat patients, because of the 
thicker skin and soft tissue, we often design several parallel puncture tunnels on the guide plate that can be used during the surgery if there is a need for adjustment. Strict design and standard operation can ensure the accuracy of a $3 \mathrm{D}$ printed personalized guide plate on the skin surface. All cases completed in our clinics were carried out according to the preoperation plan; the results were verified by intraoperative $\mathrm{X}$-ray fluoroscopy and postoperative histopathology.

Although modern medicine already has computer navigation, robot, and other directional equipment [15], for assisting core decompression surgery and for accurate drill of the core decompression, most hospitals and patients cannot afford the high cost of the directional equipment. Thus, orthopedic surgeons still rely on hand punctures and $\mathrm{X}$-rays to repeatedly adjust the position. When the patient has small necrosis focus areas or a necrosis focus positioned in a special location, inaccurate multiple drills by the surgeon may ensure. As a new technology, 3D printing personalized guide plate positioning has the advantages of low price, accurate positioning during surgery, and no damage to healthy tissue, which improves surgical efficiency, making the use of $3 \mathrm{D}$ printing effective, and this technology overcomes the deficiencies of the traditional surgical methods.

To sum up, digital design and 3D printing technology are convenient for the femoral head core decompression procedure. They offer accuracy, safety, affordability, and suitability for all levels of medical institutions and is thus worthy to be promoted for use in clinical practice.

\section{Data Availability}

The readers can access the data supporting the conclusions of this study.

\section{Conflicts of Interest}

All the authors have no conflict of interest.

\section{Acknowledgments}

This work was supported by the Dalian Science and Technology Innovation Fund Project (no.2020JJ27SN077), the Dalian Science and Technology Innovation Fund Project (no.2018J11CY030), and the Health Industry research Project (no.201402016).

\section{References}

[1] M. A. Mont, H. S. Salem, N. S. Piuzzi, S. B. Goodman, and L. C. Jones, "Nontraumatic osteonecrosis of the femoral head: where do we stand today?: A 5-year update," The Journal of Bone and Joint Surgery. American Volume, vol. 102, no. 12, pp. 1084-1099, 2020.

[2] H. R. Choi, M. E. Steinberg, and E. Y. Cheng, "Osteonecrosis of the femoral head: diagnosis and classification systems," Current Reviews in Musculoskeletal Medicine, vol. 8, no. 3, pp. 210-220, 2015.

[3] C. G. Zalavras and J. R. Lieberman, "Osteonecrosis of the femoral head: evaluation and treatment," The Journal of the Amer- ican Academy of Orthopaedic Surgeons, vol. 22, no. 7, pp. 455464, 2014.

[4] S. Banerjee, K. Issa, R. Pivec, B. H. Kapadia, H. S. Khanuja, and M. A. Mont, "Osteonecrosis of the hip: treatment options and outcomes," The Orthopedic Clinics of North America, vol. 44, no. 4, pp. 463-476, 2013.

[5] C. Wang, L. Zhang, T. Qin et al., "3D printing in adult cardiovascular surgery and interventions: a systematic review," Journal of Thoracic Disease, vol. 12, no. 6, pp. 3227-3237, 2020.

[6] T. Bartel, A. Rivard, A. Jimenez, C. A. Mestres, and S. Müller, "Medical three-dimensional printing opens up new opportunities in cardiology and cardiac surgery," European Heart Journal, vol. 39, no. 15, pp. 1246-1254, 2018.

[7] W. C. H. Parr, J. L. Burnard, P. J. Wilson, and R. J. Mobbs, “3D printed anatomical (bio)models in spine surgery: clinical benefits and value to health care providers," Journal of Spine Surgery, vol. 5, no. 4, pp. 549-560, 2019.

[8] T. P. Pierce, J. J. Jauregui, R. K. Elmallah, C. J. Lavernia, M. A. Mont, and J. Nace, "A current review of core decompression in the treatment of osteonecrosis of the femoral head," Current Reviews in Musculoskeletal Medicine, vol. 8, no. 3, pp. 228232, 2015.

[9] P. Hernigou and F. Beaujean, "Treatment of osteonecrosis with autologous bone marrow grafting," Clinical Orthopaedics and Related Research, vol. 405, pp. 14-23, 2002.

[10] M. T. Houdek, C. C. Wyles, J. R. Martin, and R. J. Sierra, "Stem cell treatment for avascular necrosis of the femoral head: current perspectives," Stem Cells and Cloning: Advances and Applications, vol. 7, pp. 65-70, 2014.

[11] E. Larson, L. C. Jones, S. B. Goodman, K. H. Koo, and Q. Cui, "Early-stage osteonecrosis of the femoral head: where are we and where are we going in year 2018?," International Orthopaedics, vol. 42, no. 7, pp. 1723-1728, 2018.

[12] L. Lin, Y. Jiao, X. G. Luo et al., "Modified technique of advanced core decompression for treatment of femoral head osteonecrosis," World Journal of Clinical Cases, vol. 8, no. 13, pp. 2749-2757, 2020.

[13] A. K. Aggarwal, K. Poornalingam, A. Jain, and M. Prakash, "Combining platelet-rich plasma instillation with core decompression improves functional outcome and delays progression in early-stage avascular necrosis of femoral head: a 4.5- to 6year prospective randomized comparative study," The Journal of Arthroplasty, 2020.

[14] J. Beckmann, J. Goetz, H. Baethis, T. Kalteis, J. Grifka, and L. Perlick, "Precision of computer-assisted core decompression drilling of the femoral head," Archives of Orthopaedic and Trauma Surgery, vol. 126, no. 6, pp. 374-379, 2006.

[15] F. Wang, J. Zhu, X. Peng, and J. Su, "The application of 3D printed surgical guides in resection and reconstruction of malignant bone tumor," Oncology Letters, vol. 14, no. 4, pp. 4581-4584, 2017. 